\title{
Validación de la prueba de Tiras cómicas para evaluar la empatía en una muestra de población mexicana
}

\author{
Dania Nimbe Lima-Sánchez * ${ }^{*}$ Xóchitl Duque-Alarcón², Fiacro Jiménez-Ponce³, Rafael Salín-Pascual, \\ Francisco Morales-Carmona ${ }^{5}$ y Arturo Ongay Peréz ${ }^{6}$ \\ ${ }^{1}$ Departamento de Informática Biomédica, Departamento de Psiquiatría y Salud Mental, Facultad de Medicina, Universidad Nacional Autónoma de \\ México (UNAM); ${ }^{2}$ Unidad de Neuropsiquiatría Tlatelolco, Instituto de Seguridad y Servicios del Estado; ${ }^{3}$ Hospital General de México Eduardo \\ Liceaga; ${ }^{4}$ Departamento de Psiquiatría y Salud Mental, Facultad de Medicina, UNAM; ${ }^{5}$ Departamento de Psicología Instituto Nacional de Perinatología \\ Isidro Espinosa de los Reyes; ${ }^{6}$ Clínica de Intervención Familiar Hospital Psiquiátrico Fray Bernardino Álvarez. Ciudad de México, México
}

\section{Resumen}

Antecedentes: La empatía es la capacidad de un individuo para entender al otro desde el punto de vista cognitivo y emocional. La empatía es un constructo complejo que representa un reto en su evaluación, por lo cual es necesario contar con instrumentos validados en población mexicana. Objetivo: Validación de la prueba de Tiras cómicas para evaluar empatía. Método: Muestreo por conveniencia, se midió la consistencia interna, la validez convergente con el coeficiente de empatía, la validez de constructo con análisis factorial y la capacidad de discriminar entre sujetos con déficit de empatía por psicopatología con controles por medio de una curva Receiver Operating Characteristic (ROC) por sus siglas en inglés. Resultados: La escala de Tiras cómicas se evaluó en 86 controles, 19 pacientes con esquizofrenia, $\alpha$ de Cronbach $=0.894$. La correlación con el coeficiente de empatía fue de 0.88 , con una sensibilidad del $81.3 \%$ y la especificidad del $38 \%$. Conclusiones: Se validó la prueba de Tiras cómicas con adecuados parámetros psicométricos.

PALABRAS CLAVE: Empatía. Esquizofrenia. Validación. Prueba. México.

\section{Abstract}

Introduction: Empathy is the ability of an individual to understand it from another cognitive and emotional point of view. Empathy is a complex that represents a retouch in its evaluation, so, it is necessary to have instruments validated in the Mexican population. Objective: Validation of the Comic Strip Test to evaluate empathy. Method: Sampling for convenience, based on internal consistency, convergent validity with the Empathy coefficient, construct validity in the factorial analysis, and the ability to discriminate between subjects with lack of empathy (psychopathology) and the control subjects through a curve ROC. Results: The comic strips test was evaluated in 86 controls, 19 patients with schizophrenia (Cronbach's alpha $=0.894$ ). The correlation with the empathy coefficient was 0.88 , with sensitivity of $81.3 \%$ and specificity of $38 \%$. Conclusions: The Comic Strip Test was validated with parametric psychometric parameters.

KEY WORDS: Empathy. Schizophrenia. Validation. Test. Mexico.

Correspondencia:

*Dania Nimbe Lima-Sánchez

E-mail: danianimbe@ hotmail.com
Fecha de recepción: 06-03-2019

Fercha de aceptación: 19-03-2019

DOI: 10.24875/GMM.19005129
Gac Med Mex. 2019;155(Suppl 1):S45-S49 Disponible en PubMed www.gacetamedicademexico.com 


\section{Introducción}

La habilidad para comunicarnos, entender las intenciones y sentimientos de los otros es una herramienta vital para las actividades sociales. Una de estas habilidades es la empatía, considerada como la capacidad de entender y dar una respuesta adecuada a las emociones, pensamientos y conductas de la otra persona, poniéndose en el lugar del otro'. En la mayor parte de los estudios sobre empatía se han establecido componentes que se relacionan con áreas del contexto social, reconocimiento de emociones y de una respuesta adecuada al ambiente ${ }^{2}$. La carencia de empatía está asociada con un pobre funcionamiento, y se observa en pacientes con trastornos psiquiátricos como en el autismo y la esquizofrenia ${ }^{3}$. En la esquizofrenia, la ausencia de empatía se ha descrito como un síntoma frecuente del trastorno, pudiéndose identificar en fases prodrómicas y llegando a ser un factor discriminante entre los pacientes y los sujetos sanos $^{4-6}$; adicionalmente la ausencia de empatía no mejora con el tratamiento farmacológico estándar, pero al parecer la oxitocina puede ser un candidato para el tratamiento?

La evaluación de la empatía es compleja debido a los componentes antes mencionados. Se han propuesto múltiples pruebas, que se pueden dividir en dos modalidades: los autorreportes y la respuesta a la presentación de un paradigma. En el caso de los autorreportes, una de las pruebas más utilizadas es el coeficiente de empatía, diseñada por Baron-Cohen $^{8}$. Esta prueba fue diseñada con base en la teoría de sistematización, bajo una visión integradora de tres componentes: empatía cognitiva, reactividad emocional y herramientas sociales. Muestra adecuadas propiedades psicométricas, con una consistencia interna de $0.85^{9,10}$. Además, se han reportado investigaciones en las que se muestra su utilidad para evaluar el espectro autista, la anorexia y en los pacientes con esquizofrenia, correlacionándose con hallazgos genéticos ${ }^{11}$. Esta escala considera 60 preguntas de las cuales 28 son ítems indicadores, mientras que el resto son de control, con respuestas tipo Likert que tienen las siguientes opciones: de acuerdo, acuerdo parcial, en desacuerdo parcial y totalmente en desacuerdo. Fue planificada para tener una aplicación clínica. La validación de la prueba en población mexicana se realizó en 200 sujetos de entre 18 y 25 años capaces de comprender escalas, obteniendo una consistencia interna de 0.81 y una fiabilidad test-retest $r=0.80$; la media de la calificación fue de 41.1 puntos $^{12}$.

Por otro lado, en las evaluaciones que se basan en la aplicación de un paradigma, se ha demostrado la utilidad de la prueba de las Tiras cómicas propuestas por Vollm ${ }^{12}$, que posteriormente fue modificada por $\mathrm{Lee}^{13}$. Esta prueba toma en cuenta los siguientes componentes: empatía cognitiva, empatía emocional, empatía inhibitoria y causalidad física como sección de control. Cada condición se califica con 12 tiras cómicas que ilustran diferentes escenarios. Los dibujos utilizados en este estudio se basaron en los que originalmente desarrollaron Völlm, et al. ${ }^{12}$, y que posteriormente fueron modificados por Lee ${ }^{13}$. Este último autor amablemente nos proporcionó la prueba de las Tiras cómicas en una comunicación personal. Cada tira cómica se compone de tres cuadros que ilustran una interacción entre dos personajes. El personaje principal o protagonista, quien se muestra en violeta, tiene una respuesta empática con el otro personaje respondiendo a la intención o la emoción de este último. Los cuatro tipos de dibujos animados se validaron por Lee en el 2010, en un estudio piloto con 16 sujetos sanos entre 24 y 35 años; en sus resultados no se reportó sensibilidad y especificidad ${ }^{13}$.

No obstante los antecedentes mencionados, aún no hay un consenso en la forma de evaluar la empatía y la prueba de Tiras cómicas no ha sido validada ni utilizada en México.

El objetivo de este trabajo es obtener las propiedades psicométricas de la prueba de Tiras cómicas en una muestra de sujetos mexicanos.

\section{Métodos}

La selección de la muestra fue por conveniencia. Los participantes en este estudio eran estudiantes de medicina de la Universidad Nacional Autónoma de México, estudiantes de rehabilitación y población abierta. La aplicación del instrumento se realizó por parte de los investigadores del estudio de manera individual, en un área destinada para ello (consultorio médico o aula).

Para examinar la sensibilidad para detectar individuos que se espera que muestren un déficit en empatía, se evaluaron pacientes con esquizofrenia que fueron diagnosticados de manera formal por psiquiatras, utilizando los criterios diagnósticos del de la Clasificación Internacional de Enfermedades (CIE), Versión 10. en el Hospital Psiquiátrico Fray Bernardino Álvarez. Posteriormente se calificó a los sujetos 
de cada grupo pareándolos por edad y sexo contra el grupo control.

Para medir la validez de constructo se realizó inicialmente una prueba de esfericidad de Bartlett, que evalúa si los datos de cada prueba se comportaban de forma esférica y si pudiera realizarse un análisis factorial. Para evaluar la validez convergente se empleó el coeficiente de empatía de Baron-Cohen.

La consistencia interna se obtuvo por medio del cálculo del $\alpha$ de Cronbach. La confiabilidad test-retest se realizó en 80 sujetos. Finalmente, se construyó una gráfica de curvas ROC para medir la sensibilidad y especificidad.

El protocolo de estudio fue aprobado por los Comités de Investigación y de Ética del Hospital Psiquiátrico "Fray Bernardino Álvarez". En todos los casos se realizó el proceso de consentimiento informado, la identidad de los sujetos de investigación se manejó bajo estándares de confidencialidad.

\section{Resultados}

Se realizó primero una prueba piloto en diez sujetos sanos para observar comprensión y aplicabilidad; posteriormente se aplicó a 105 sujetos de los cuales 86 eran estudiantes de medicina, técnicos en rehabilitación y acompañantes de pacientes (55 mujeres y 31 hombres); 19 sujetos fueron pacientes esquizofrénicos (10 mujeres y 9 hombres) que se encontraban en fase de remisión internados en el Hospital Psiquiátrico Fray Bernardino Álvarez (Tabla 1). Se realizó una comparación entre mujeres y hombres con la prueba $t$ de Student para muestras independientes y no se encontraron diferencias significativas ( $\mathrm{t}: 0.455 ; \mathrm{p}=0.650$ ).

La consistencia interna de la prueba fue $\alpha=0.843$. Se evaluó la validez convergente con el coeficiente de empatía propuesto por Baron-Cohen, con el cual se obtuvo una correlación $r=0.88(p=0.0001)$; además se evaluó la consistencia de la escala a los dos meses de su aplicación en una submuestra de 80 sujetos, obteniéndose una correlación $r=0.572$ $(p=0.03)$. Se realizó la prueba de esfericidad de Bartlett $(p=0.406)$ y la prueba de adecuación muestral de Kaiser-Mayer-Olkin ( $\chi^{2:}$ 2012.6; $\left.p=0.001\right)$. La matriz de componentes rotados mostró una tendencia a correlacionar los factores 1 y 2 , pero el resto de los factores mostró una diferenciación adecuada entre cada uno de ellos. Con respecto al análisis factorial, la mayor parte de los ítems de la prueba de Tiras cómicas mostró una tendencia a encontrase en el
Tabla 1. Datos demográficos de la muestra divididos por género. En la tabla se indica la distribución de la muestra por edad y sexo, donde se observa un mayor porcentaje de mujeres

\begin{tabular}{|l|c|c|}
\hline \multirow{2}{*}{ Variable } & \multicolumn{2}{|c|}{ Tiras cómicas } \\
\cline { 2 - 3 } & $\begin{array}{c}\text { Controles } \\
n(\%)\end{array}$ & $\begin{array}{c}\text { Esquizofirénicos } \\
n(\%)\end{array}$ \\
\hline $\begin{array}{c}\text { Sexo } \\
\text { Mujer } \\
\text { Hombre }\end{array}$ & 55 & 10 \\
\hline Edad en años (DE) & 31 & 9 \\
\hline
\end{tabular}

DE: desviación estándar

Tabla 2. Comparación de los resultados de las Tiras cómicas entre pacientes con esquizofrenia y controles. En esta tabla observamos la comparación entre controles y pacientes pareados por edad y sexo en las dos pruebas, mostrando diferencias en todos los parámetros de la prueba de Tiras cómicas

\begin{tabular}{|l|c|c|c|c|}
\hline Variable & Pacientes & Controles & Prueba t & Significanciáa \\
\hline Tiras cómicas & & & & \\
\hline Empatía cognitiva & $8.8(2.4)$ & $10.7(1.2)$ & -2.89 & 0.006 \\
\hline Empatía emocional & $7.05(1.7)$ & $10.38(1.6)$ & -6.08 & 0.0001 \\
\hline Empatía inhibitoria & $9(2.1)$ & $10.1(1.8)$ & -2.018 & 0.09 \\
\hline Causalidad física & $8.8(1.8)$ & $11.11(1.4)$ & -5.44 & 0.0001 \\
\hline Total Tiras cómicas & $33.84(6.3)$ & $42.38(5.4)$ & -6.38 & 0.0001 \\
\hline
\end{tabular}

factor uno, hubo dos ítems, el 42 y el 46 que no mostraron correlación con ningún factor.

\section{Curvas ROC}

La prueba de Tiras cómicas mostró un punto de corte de 41.5 de un total de 48 , obteniéndose una sensibilidad de 0.813 y una especificidad de 0.338 (área: 0.851; $p=0.0001$ ) (Fig. 1).

Se parearon los pacientes por grupos de edad y sexo con los controles, todos los pacientes mostraron resultados significativamente menores en todas las secciones de la prueba (Tabla 2).

\section{Discusión}

La empatía es una habilidad que se utiliza en la vida diaria, ya que los seres humanos somos altamente sociables y es una herramienta fundamental para la interacción; pero a pesar de su importancia todavía hay una falta de consenso sobre su definición por la complejidad que implica ${ }^{14}$. Esto se refleja en las múltiples áreas anatómicas en el cerebro que se activan durante su funcionamiento, y que tienen un componente genético muy importante ${ }^{15}$; además podemos 


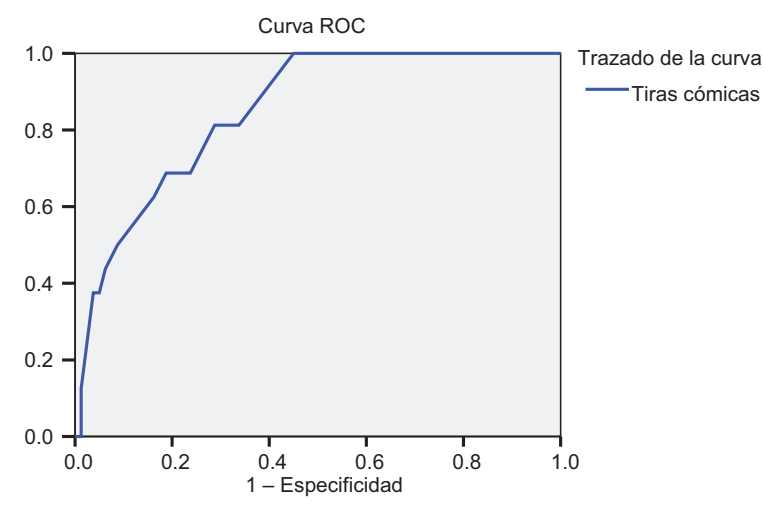

Figura 1. Curva ROC de la prueba de Tiras cómicas y el coeficiente de empatía. En esta gráfica 7 se observa la curva ROC, obteniendo un valor de la curva significativo.

encontrar alteraciones en diferentes estados patológicos, como es el caso de la esquizofrenia, donde causa una disfunción global y que puede estar asociada a procesos metacognitivos ${ }^{4}$.

En los resultados de la validación de las Tiras cómicas podemos observar que desde el punto de vista psicométrico los valores obtenidos en el estudio fueron parecidos a los referidos por $\mathrm{Lee}^{13}$, lo que la convierte en una herramienta útil y con menores sesgos que las pruebas basadas en autoinformes, útil para evaluar a pacientes con esquizofrenia. Generalmente los autorreportes pueden tener un menor poder discriminante cuando se utiliza en pacientes y población general, por lo que las pruebas que se presentan por medio de un paradigma son más eficaces al replicar de forma fehaciente un escenario real; esto se ha demostrado en estudios que muestran que las pruebas autoinformadas pueden estar fuertemente influenciadas por las normas sociales y expectativas de género. En este aspecto coincidimos, nuestro estudio no mostró diferencias en los niveles de empatía, ya que generalmente se obtienen mayores niveles de empatía en las mujeres cuando se utilizan pruebas de autorreporte ${ }^{16}$. El análisis factorial sugiere que el ítem 42 y el 46 podrían eliminarse de la prueba. La revisión de estos dos ítems lleva a concluir que el 46 es un control de causalidad física que podría no ser claro, por lo que se puede plantear proponer otro paradigma. El ítem 42 es un ejemplo de empatía inhibitoria de dos amigos, en el cual uno se encuentra intoxicado por alcohol y el sujeto tiene que decidir si lo deja conducir o no, este ejemplo nos puede llevar a la posibilidad de estar ante un dilema que por la edad de la muestra, en su mayoría jóvenes, muestren menores niveles de cuidado en este aspecto $^{17}$, por lo que sería deseable replicar este análisis en una muestra con edades más heterogéneas.
Nuestro estudio presenta algunas limitaciones, como el tamaño pequeño de la muestra y su selección, que no fue aleatoria, lo que podría ser un factor de confusión por el que no obtuviéramos todo el espectro de severidad, lo que podría sesgar los resultados en comparación con la población general. Para futuros estudios sería recomendable la creación de instrumentos para evaluar empatía basadas en simulaciones o prueba mixtas que puedan abordar el componente cognitivo o las herramientas sociales por medio de estímulos visuales y escenarios, con el fin de realizar un abordaje más adecuado, además de evaluar las maniobras de intervención que puedan mejorar esta capacidad, como las basadas en metacognición ${ }^{18}$.

Podemos concluir que el instrumento mostró adecuadas propiedades psicométricas para utilizarse en muestras mexicanas, sin embargo, debe ampliarse la muestra para mejorar su validez.

\section{Agradecimientos}

Agradecemos el trabajo del Dr. Rodrigo Figueroa Vera por su ayuda para la aplicación de instrumentos.

Agradecemos a la Dra. María del Carmen Lara Muñoz su asesoría y guía en este proceso.

\section{Financiación}

Los autores declaran carecer de fuente de financiamiento.

\section{Conflicto de intereses}

Los autores declaran que no existen conflictos de intereses.

\section{Confidencialidad de los datos}

Los autores declaran que en este artículo no aparecen datos de pacientes.

\section{Bibliografía}

1. Greenberg DM, Warrier V, Allison C, Baron-Cohen S. Testing the Empathizing-Systemizing theory of sex differences and the Extreme Male Brain theory of autism in half a million people. Proc Natl Acad Sci U S A. 2018;115:12152-7.

2. Coll MP, Viding E, Rutgen M, Silani G, Lamm C, Catmur C, et al. Are we really measuring empathy? Proposal for a new measurement framework. Neurosci Biobehav Rev. 2017;83:132-9.

3. Koelkebeck K, Kuegler L, Kohl W, Engell A, Lencer R. Social cognition in schizophrenia: The role of mentalizing in moral dilemma decision-making. Compr Psychiatry. 2018;87:171-8.

4. Kállai J, Rózsa S, Hupuczi E, Hargitai R, Birkás B, Hartung I, et al. Cognitive fusion and affective isolation: Blurred self-concept and empathy deficits in schizotypy. Psychiatry Res. 2019;271:178-86. 
5. Atoui M, El Jamil F, El Khoury J, Doumit M, Syriani N, Khani M, et al. The relationship between clinical insight and cognitive and affective empathy in schizophrenia. Schizophr Res Cogn. 2018;12:56-65.

6. Grant N, Lawrence M, Preti A, Wykes T, Cella M. Social cognition interventions for people with schizophrenia: a systematic review focussing on methodological quality and intervention modality. Clin Psychol Rev. 2017; $56: 55-64$.

7. Halverson T, Jarskog LF, Pedersen C, Penn D. Effects of oxytocin on empathy, introspective accuracy, and social symptoms in schizophrenia: A 12-week twice-daily randomized controlled trial. Schizophr Res. 2019;204:178-82

8. Baron-Cohen S, Wheelwright $\mathrm{S}$. The empathy quotient: an investigation of adults with Asperger syndrome or high functioning autism, and normal sex differences. J Autism Dev Disord. 2004;34:163-75.

9. Allison C, Baron-Cohen S, Wheelwright SJ, Stone MH, Muncer SJ. Psychometric analysis of the Empathy Quotient (EQ). Pers Individ Dif. 2011;51:829-35.

10. Muncer SJ, Ling J. Psychometric analysis of the Empathy Quotient (EQ) scale. Pers Individ Dif. 2006:40:1111-9.

11. Warrier V, Toro R, Chakrabarti B, Borglum AD, Grove J, Hinds DA, et al. Genome-wide analyses of self-reported empathy: correlations with autism, schizophrenia, and anorexia nervosa. Translational psychiatry. 2018;8:35
12. Vollm BA, Taylor AN, Richardson P, Corcoran R, Stirling J, McKie S, et al. Neuronal correlates of theory of mind and empathy: a functional magnetic resonance imaging study in a nonverbal task. Neuroimage. 2006;29:90-8.

13. Lee SJ, Kang DH, Kim CW, Gu BM, Park JY, Choi CH, et al. Multi-level comparison of empathy in schizophrenia: an fMRI study of a cartoon task. Psychiatry Res. 2010;181:121-9.

14. Batchelder L, Brosnan M, Ashwin C. The development and validation of the Empathy Components Questionnaire (ECQ). PLoS One. 2017;12:e0169185.

15. Heyes C. Empathy is not in our genes. Neurosci Biobehav Rev. 2018;95:499-507.

16. Baez S, Flichtentrei D, Prats M, Mastandueno R, Garcia AM, Cetkovich M, et al. Men, women. who cares? A population-based study on sex differences and gender roles in empathy and moral cognition. PLoS One. 2017; 12:e0179336.

17. Worly B, Verbeck N, Walker C, Clinchot DM. Burnout, perceived stress, and empathic concern: differences in female and male Millennial medical students. Psychol Health Med. 2019;24:429-38.

18. Bonfils KA, Lysaker PH, Minor KS, Salyers MP. Metacognition, personal distress, and performance-based empathy in schizophrenia. Schizophr Bull. 2019;45:19-26. 